\title{
Relevance versus reliability of accounting information with unlimited and limited commitment
}

\author{
Barbara Schöndube-Pirchegger ${ }^{1}$. \\ Jens Robert Schöndube ${ }^{2}$ (1)
}

Received: 20 May 2016/ Accepted: 19 June 2017/Published online: 5 July 2017

(C) The Author(s) 2017. This article is an open access publication

\begin{abstract}
We consider a two-period LEN-type agency problem. The principal needs to implement one out of two accounting systems. One emphasizes relevance, the other reliability. Both systems produce identical inter-temporally correlated signals. The relevant system reports an accounting signal in the period in which it is produced. The reliable system reports a more precise signal, but with a one period delay. Accounting information is contractible only if it is reported within the two-period horizon of the game. Accordingly, accounting information produced in the second period becomes uncontractible with the reliable system in place. Non-accounting information needs to be used for contracting to provide any second period incentives at all. We derive optimal compensation contracts in a full and in a limited commitment setting. With full commitment, the reliable system trades-off more precise first and less precise second-period contractible information, as compared to the relevant system. If the reduction of noise in the accounting signals is strong and the distortion in the non-accounting measure is weak, the reliable system is preferred. With limited commitment we identify a similar trade-off if intertemporal correlation of the signals is negative. If it is positive, this tradeoff might reverse: The reliable system is possibly preferred if noise reduction is small and the non-accounting measure is heavily distorted. Noisiness in performance
\end{abstract}

We acknowledge helpful comments from Joachim Gassen (the editor), two anonymous reviewers, Christian Hofmann, Sandra Kronenberger, Konrad Lang, Carolin Mauch, and from conference/seminar participants at Berlin (2013), Graz (2015) and Tallinn (EAA annual conference 2014).

Jens Robert Schöndube

schoendube@controlling.uni-hannover.de

Barbara Schöndube-Pirchegger

barbara.schoendube@ovgu.de

1 Otto-von-Guericke University Magdeburg, Universitätsplatz 2, 39106 Magdeburg, Germany

2 Leibniz Universität Hannover, Königsworther Platz 1, 30167 Hannover, Germany 
measures then serves as a commitment device. It reduces otherwise overly high powered incentives and thus benefits the principal.

Keywords Accounting information - Agency-problem - Limited commitment ·

Timeliness

JEL Classification M4 $\cdot$ D8 $\cdot$ C7

\section{Introduction}

In this paper we juxtapose two well-known accounting concepts: relevance and reliability. Both are anchored not only in the IASB Conceptual Framework, but also in US-GAAP and various other (local) sets of standards. Relevance requires that accounting information is capable of affecting decisions made by its users. This relates to timeliness, comparability, and understandability. Reliability refers to undistorted complete information that is free from errors. Verifiability and credibility are important issues here. Unfortunately, both concepts are not necessarily consistent. At least for some reporting items the need to ensure reliability of information may result in delayed publication, which in turn reduces relevance (see, e.g., Alexander and Nobes 2013, p. 43). For such items, users as well as standard setting bodies need to stress either one or the other: relevance or reliability.

Contemplating recent developments in accounting standard setting and practice, an increased number of firms seems to lean towards relevance rather than reliability. ${ }^{1}$ Multiple factors drive this trend. For once, the IASB clearly focuses on providing decision-relevant information to investors ${ }^{2}$ and increasingly allows/ requires timely recognition. This includes fair value accounting, revaluation of assets, and revenue recognition based on percentage of completion. Second, an increasing number of firms actually apply IFRS, rather than national GAAP. They do so either voluntarily or forced by national and/or EU-regulations. ${ }^{3}$ Finally, local standard setters, e.g., in Germany, revised their set of national standards aiming at harmonization and thus aligning them to international regulations. ${ }^{4}$

Whether a focus on relevance rather than reliability renders accounting information more useful, however, is far from obvious. To tackle this question at all, we need to acknowledge that accounting information is used for different purposes, in particular valuation and contracting purposes. Since an accounting system that is preferred for valuation is not necessarily preferred for contracting and v.v., any analysis needs to specify the considered purpose. ${ }^{5}$

In this paper, we pursue the question of "relevance or reliability" taking a contracting perspective. Using a stylized model, we contrast both concepts

\footnotetext{
${ }^{1}$ E.g., Andre and Filip (2012) find evidence not only for reduced emphasis on reliability but for a reduction in conservatism in general in financial statements of EU-countries.

2 See, e.g., Preface to IFRS.

3 See Pacter (2015).

${ }^{4}$ Such as via the BilMoG in 2009.

5 See Gjesdal (1981).
} 
assuming that two sets of standards are available. One set emphasizes relevance and the other emphasizes reliability. Relevance in our model translates into early reporting of the information, in the sense of timeliness. Reliability is tantamount to late, but less noisy reporting, in order to ensure a high level of credibility.

We consider a two-period principal-agent relationship. The agent performs an effort in both periods and the principal aims at providing incentives via an appropriate compensation contract. One out of two different types of accounting or reporting systems is possibly implemented. Both systems produce identical information at the end of each period. Signals are informative with respect to the agent's effort. Inter-period correlation of the signals is present. The first system reports accounting information immediately, that is, in the period it is produced. The second system delays reporting of each signal by one period reflecting strong emphasis on reliability. Later reporting goes along with less noisy signals. In what follows, we denote the former system the timely or early information system, and the latter the late information system.

To provide an example for our presumed setting, assume that annual revenues are used for performance measurement. Revenue, e.g. from sales of some good, is recorded when it is realized. Typical indicators for realization are the transfer of significant risks and control over the good to the buyer. In addition, the amount to be recorded needs to be reliably measurable and an actual flow of benefits to the firm has to be probable. Whether these indicators are fulfilled at some point in time, in many cases is a matter of judgement. Eventually, whether recognition occurs sooner or later depends on how much emphasis is put on relevance, as opposed to reliability. In terms of our story, the timely system records revenue earlier. The amount reported, however, is noisy. The late system, in contrast, demands a higher level of reliability w.r.t. the amount recorded that is only generated over time. As a result, recognition occurs with some delay but future benefits of the amount recorded can be safely assumed.

Reporting is a necessary precondition for contractability in our setting, as it renders information verifiable by a third party. Beyond that, delay of reporting critically affects contractability. To see that, consider a signal that is reported sometime after the agent has left the firm in a distant future. In terms of our above example, revenue from some long-term service or construction contract is possibly realized only with considerable delay. Such a signal becomes practically non-contractible as waiting for its realization is unsuitable. We reflect this aspect in our model by assuming that information is contractible only if it becomes observable and verifiable within the two period horizon of our game. Thus, the direct effect of late information in the model is less contractible information. With the late accounting information system in place, the secondperiod accounting signal becomes unavailable for contracting, as it will not be reported throughout the game. The late accounting system, in fact, is one that provides a reduced set of performance measures for contracting, as compared to the early information system. ${ }^{6}$ We assume, however, some non-accounting

\footnotetext{
${ }^{6}$ We consider delayed reporting as the very reason for a reduced set of contractible information. The results derived, however, hold for any two types of accounting systems that provide the principal with different signals to be contracted upon, no matter what causes the unavailability of a measure in one system.
} 
measure is present in this case, such that incentive setting is generally possible in period two.

Within this structure, we contrast a full commitment and a limited commitment setting. Full commitment implies that the principal and the agent agree upon a twoperiod contract at the start of the game, which remains unchanged throughout the game. In the limited commitment setting, we consider a setting with two short-term contracts such that sequentially optimal contracting decisions apply.

With full commitment, we find that the late system is preferred to the early one only if the reduction in noise from the early to the late first period signal is sufficiently strong and if the second-period action can be controlled with sufficient precision.

However, in most real live settings, it is unlikely that contracting parties can effectively commit to stick to an ex post inefficient contract. Studying limited commitment instead, first of all, we find that the expected payoff to the principal decreases no matter which accounting system is used. Given the assumed correlation of signals over time, limited commitment causes optimal ex post but suboptimal ex ante incentives in period two. As a result, agency costs increase as opposed to full commitment.

With regard to preferability of either the early or the late system under limited commitment, our results differ depending on the presumed correlation of signals over time. If the signals are negatively correlated, our results pretty much resemble the ones from full commitment. With positive intertemporal correlation they differ qualitatively. It turns out that in some settings the late accounting system becomes optimal, when the reduction in noise from the early to the late system is small, and the early system dominates if noise reduction is large.

Whether intertemporal correlation of accounting measures is more likely to be positive or negative depends on the situation at hand. Using the example of revenues once again, correlation is likely to differ with the specifics of the goods considered. For convenience goods we would expect intertemporal correlation to be positive, as larger sales in one period trigger repeated purchases in future periods. In contrast, specialty goods that are purchased in one period might not be purchased in the next and v.v., implying that a negative intertemporal correlation should be expected. More generally, accounting accruals or even accounting errors are likely to reverse sometime in the future and thus induce a negative correlation. If, on the other hand, underlying economic effects such as a persistent increase in demand are present, this favors positive correlation. Ultimately, both, accruals and persistent effects, might be simultaneously relevant and positive or negative correlation hinges on which effect is stronger. ${ }^{7}$

To sum up our main results, with limited commitment and negative intertemporal correlation (and also with full commitment), the results are almost what we would expect: Early recognition is helpful and a late system is preferred only if the increase in precision (decrease in noise) is sufficiently large. However, with positive correlation, the opposite might be true. Intuitively, persistent noise counteracts overly high ex post incentives that are present with positive correlation, and in this sense serves as a commitment to lower incentives.

\footnotetext{
$\overline{{ }^{7} \text { See, e.g., Christensen et al. (2005) for a more detailed discussion. }}$
} 
It follows from these findings that even from a pure contracting perspective neither relevance nor reliability can be identified as the uniquely preferred concept. Accordingly, we cannot derive a clear-cut recommendation to standard setters from our model. We do show, however, that both concepts are potentially beneficial. Thus, our model suggests that diversity in systems, and flexibility of firms to choose from different systems, helps to reduce overall agency costs. Having said this, we find no strong argument in favor of the aforementioned trend towards relevance.

From a management control perspective, our results show that the accounting system choice affects optimal incentive setting and contracting costs. Which system is preferable for a particular firm depends on finer details, however. In particular, the correlation of the performance measures is crucial. Under limited commitment and negative correlation, the reliable (relevant) system turns out to be more (less) beneficial the stronger the reduction in noise over time and the more precise an available non-accounting performance measure and v.v. Conversely, with highly positive correlation in accounting signals, the reliable (relevant) system is more (less) preferable if reduction in noise is low and no high-quality non-accounting measure is available for contracting in period two. Thus, from an empirical perspective, our model predicts diverse system choices across firms reflecting differences in finer details.

Our paper is naturally related to the large body of literature that examines the trade-off between relevance and reliability. Many of those papers, however, do so rather indirectly, e.g., considering fair value accounting as opposed to historical cost accounting (e.g., Liang and Riedl 2014) or revenue recognition (see, e.g., Antle and Demski 1989 and Dutta and Zhang 2002). Some explicitly focus on relevance versus reliability such as Dye and Sridhar (2004), and more recently Zhang (2012). Dye and Sridhar (2004) consider an accountant who observes some pieces of information directly and receives a report on other pieces from a manager. Direct observation is considered reliable information while reported information is relevant, but less reliable. The accountant aggregates this information when setting up financial statements and can either put more emphasis on reliable or on relevant information. Dye and Shridhar (2004) analyze the effect the aggregation procedure has on the manager's incentives, the investment choices of the firm, and the firm's market value. They also oppose information aggregation to disaggregation.

Zhang (2012) investigates welfare effects of mandatory disclosure if information is more or less relevant/reliable. Allowing for voluntary disclosure to complement mandatory disclosure, he finds that reducing the latter to reliable information not only induces more voluntary reporting but also might increase welfare. We are not aware, however, of a paper that investigates the relevance and reliability trade-off with reference to timeliness of reporting, as we do in this paper. Besides, limited commitment has no role in this literature, but is crucial in our setting. Hence, our paper also relates to the literature on limited commitment. It does so in two ways: First, we strongly rely on findings from previous work on limited commitment. Specifically, we rely on Fudenberg et al. (1990), Indjejikian and Nanda (1999), and Christensen et al. (2003, 2005). Fudenberg et al. (1990) demonstrated that in the absence of long-term commitment, the equilibrium outcome is determined by sequentially rational contracting decisions. They also show that ex post efficient 
contracts may well be inefficient from an ex ante perspective. The inefficiency results in a loss in welfare that can be avoided in special cases only. Indjejikian and Nanda (1999) and Christensen et al. (2003, 2005) found that in a two-period LENsetting, limited commitment generally creates a welfare loss if performance measures are inter-temporarily correlated. ${ }^{8}$ This result directly extends to our paper.

Second, our results show some similarities to previous papers on limited commitment even though they are derived from a different setting. In particular, Indjejikian and Nanda (1999) show that aggregation of performance signals may be valuable under limited commitment. In contrast, in our setting, there is no information aggregation. Rather, the differences in noisiness of first- and secondperiod contractible information and the different timeliness of this information is crucial.

Finally, while we show in this paper that the late accounting system under certain circumstances can be used as a commitment to low incentives, SchöndubePirchegger and Schöndube (2012) show that delegation of decision rights may serve as a commitment to higher powered incentives.

\section{The model}

We consider a two-period LEN-model of repeated moral hazard. At the beginning of the first period the principal hires an agent to perform a certain task $a_{t}$ in each period $t=1,2$. The agent's total contribution to firm value is given by

$$
x=a_{1}+a_{2} .
$$

We assume that the agent's contribution to firm value is not available for contracting, e.g., because it becomes observable and contractible in a distant future only. ${ }^{9}$ In fact, throughout this paper we generally restrict contractability to measures that become observable and verifiable within the two-period horizon of our game. ${ }^{10}$

We consider two alternative accounting systems. Reporting from both systems is depicted in Fig. 1. Both systems differ with respect to the timing of reporting and the precision of the reported items. Once a piece of information is reported in the financial statements, it becomes contractible. The timely accounting system $A_{1}$ reports some signal $y_{1}$ at $t=1$ and $y_{2}$ at $t=2$. Accounting system $A_{2}$, in contrast, reports each signal with one period delay, but reported signals are less noisy. It reports signal $y_{1}^{L}$ at $t=2$ and $y_{2}^{L}$ only at $t=3$ (which is outside the contracting horizon). The second system can be regarded as a more conservative accounting

\footnotetext{
8 In contrast, in a risk-neutral setting Schöndube (2008) shows that limited commitment with short-term contracts may be beneficial if contracting parties learn about the agent's productivity.

9 See, e.g., Feltham and Xie (1994), Gibbons (2005).

10 Although contracting on measures outside the contracting horizon would be possible theoretically, in many cases it seems to be unusual to consider a contract that relies on measures that are realized after the manager has left the firm. An important argument against using post retirement measures is that their use is not sequentially optimal (see also the discussion in Sect. 5). That is, after the agent has left the firm it is always optimal for the principal to change any performance based compensation into a fixed compensation in order to perfectly insure the risk averse agent. As limited commitment is a crucial assumption of our paper we do not consider contracting on variables beyond $t=2$.
} 


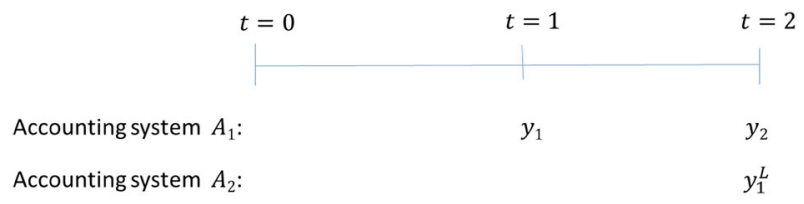

Fig. 1 Accounting systems $A_{1}$ and $A_{2}$

system that requires a higher degree of reliability in order to report a certain type of information. Note, however, that the information produced under both accounting systems is identical. Although $y_{1}$ is not reported in the financial statements under accounting system $A_{2}$, it is produced by the system and observed by the contracting parties at $t=1$. The same is true for $y_{2}$.

We define the accounting signals as follows:

$$
y_{t}^{L}=a_{t}+\varepsilon_{t}
$$

and

$$
y_{t}=y_{t}^{L}+\theta_{t}
$$

$y_{1}^{L}$ and $y_{2}^{L}$ (and $y_{1}$ and $y_{2}$ as well) depend on the agent's effort $a_{t}$ in the respective period. $\varepsilon_{1}$ and $\varepsilon_{2}$ are normally distributed noise terms with mean zero and variance $\sigma_{\varepsilon}^{2}$. Furthermore we assume that $\operatorname{corr}\left(\varepsilon_{1}, \varepsilon_{2}\right)=\lambda . \theta_{1}$ and $\theta_{2}$ are normally distributed random variables with zero means and variance $\sigma_{\theta}^{2} . \theta_{1}$ and $\theta_{2}$ are uncorrelated and they are also not correlated to $\varepsilon_{t}$. Thus, $y_{t}$ in our model is a mean preserving spread of $y_{t}^{L}$.

Without loss of generality we define $\sigma_{\varepsilon}^{2}=\gamma \sigma^{2}$ and $\sigma_{\theta}^{2}=(1-\gamma) \sigma^{2}$ for $\gamma \in[0,1]$. Doing so, $\gamma$ determines the reduction in variance from early to late reporting. In the extreme, $\gamma=1$ results in the late signal being as noisy as the early one. For $\gamma=0$, in contrast, the late signal is completely deterministic and all variance is attributable to early reporting.

The principal implements one of the two accounting systems at $t=0$. Which one is used is either prescribed by a standard setter, who requires a more or less reliable reporting, or it may be subject to the principal's choice. In the latter case we assume that once a reporting system is implemented, it cannot be changed throughout the game.

As under the late system the accounting signal related to the manager's second period action is observed outside the contracting horizon, it is unavailable for contracting. To be able to control second-period effort at all, the firm needs to contract on an alternative non-accounting measure. We assume that such a measure exists. This is an admittedly strong assumption, but serves our needs and allows us to keep the model tractable. To account for alternative assumptions, in Sect. 5 we characterize the consequences for our results if (i) a similar measure was available in period one as well, (ii) no such measure was available in any period, and (iii) contracting on $y_{2}^{L}$ was feasible. The alternative measure is defined by

$$
P_{2}=y_{2}+\eta
$$


and could be interpreted as, e.g., a market price or cash flow measure.

$\eta$ is a normally distributed random variable with zero mean and variance $v^{2}$. It is uncorrelated to all other random variables of the model. Importantly, $P_{2}$ is determined by the second-period (early) accounting signal but it is distorted from $y_{2}$ as there is additional noise in the signal captured by $\eta$. Thus, $P_{2}$ is a garbling of $y_{2}$.

Let $\boldsymbol{a}=\left(a_{1}, a_{2}\right)$ represent the agent's effort. The agent is strictly risk averse with utility $U_{A}=-\exp (-r(S-C(\boldsymbol{a})))$. Here $S$ denotes the agent's compensation, $C(\boldsymbol{a})=\frac{a_{1}^{2}+a_{2}^{2}}{2}$ is his personal cost from providing effort, and $r>0$ is the agent's risk aversion coefficient. We restrict attention to incentive contracts that are linear in the performance measures. This assumption combined with exponential utility and normality leads to the well-known LEN-specification. The agent's preferences can be represented by

$$
C E(S, \boldsymbol{a})=E(S)-C(\boldsymbol{a})-\frac{r}{2} \operatorname{Var}(S) .
$$

We normalize the certainty equivalent of the agent's reservation utility to zero.

The principal is risk neutral. She chooses performance measures and optimal contracting coefficients to maximize her expected net outcome $U=E(x-S)$.

With respect to the agent's compensation contract, we have to distinguish $A_{1}$ and $A_{2}$ : Under $A_{1}$ the compensation contract is defined as

$$
S^{A_{1}}=f+s_{1} y_{1}+s_{2} y_{2}+z_{2} P_{2} .
$$

Under $A_{2}$ the compensation contract becomes

$$
S^{A_{2}}=f+s y_{1}^{L}+z_{2} P_{2} \text {. }
$$

Here $f$ denotes a fixed payment and $s, s_{1}, s_{2}$ and $z_{2}$ are incentive coefficients. Since under $A_{2}$ the accounting report $y_{1}^{L}$ is not available before $t=2$, we denote the bonus coefficient on $y_{1}^{L} s$ rather than $s_{1}$.

\section{Full commitment solutions}

In this section we characterize the full commitment setting. Both contracting parties can commit to a long-term two-period contract that cannot be renegotiated after it has been signed. The general optimization problem of the principal under full commitment can be stated as follows with $S \in\left\{S^{A_{1}}, S^{A_{2}}\right\}$ :

$$
\begin{gathered}
\max _{S} U=E(x-S) \\
\text { s.t. } C E(S, \boldsymbol{a}) \geq 0 \\
\boldsymbol{a}=\underset{\boldsymbol{a}^{\prime}}{\operatorname{argmax}} C E\left(S, \boldsymbol{a}^{\prime}\right)
\end{gathered}
$$

The risk-neutral principal maximizes her net return subject to two conditions. The individual rationality constraint (IR) is binding at the optimum and ensures that the agent accepts the contract. Further, the agent chooses his actions in order to maximize personal welfare. This is reflected in the incentive compatibility constraint (IC). 
We start by assuming that the accounting system $A_{1}$ has been implemented.

Lemma 1 The optimal full commitment incentive contract, given that the early system $\mathrm{A}_{1}$ is used, contains

$$
s_{1}^{*}=\frac{1}{1+r \sigma^{2}(1+\gamma \lambda)}=s_{2}^{*}, z_{2}^{*}=0 .
$$

Equilibrium surplus is

$$
U^{*}=\frac{1}{1+r \sigma^{2}(1+\gamma \lambda)} \text {. }
$$

Proof All proofs are relegated to the Appendix.

As $P_{2}$ is a garbling of $y_{2}$ it will never be used in a contract when $y_{2}$ is available. Accordingly, $z_{2}^{*}=0$ in the optimal contract under $A_{1}$. If $y_{1}$ and $y_{2}$ are negatively correlated, that is $\lambda=\operatorname{corr}\left(\varepsilon_{1}, \varepsilon_{2}\right)<0$, an insurance effect is present, that reduces the risk premium paid to the agent. With positive correlation, in contrast, the variance of the agent's compensation increases due to the covariance effect. Thus, increasing $\lambda$ rises the risk premium to be paid and in turn reduces the optimal incentive rates $s_{1}$ and $s_{2}$ and the corresponding optimal surplus.

With the late accounting information system $A_{2}$, optimal incentive rates are stated in Lemma 2 below:

Lemma 2 The optimal full commitment incentive contract under the late system $A_{2}$ uses

$$
\begin{aligned}
s^{* *} & =\frac{1+r \sigma^{2}(1-\gamma \lambda)+r v^{2}}{v^{2} r\left(1-r \sigma^{2} \gamma\right)+1+r \sigma^{2}(1+\gamma)+r^{2} \sigma^{4} \gamma\left(1-\gamma \lambda^{2}\right)}, \\
z_{2}^{* *} & =\frac{1+r \sigma^{2} \gamma(1-\lambda)}{v^{2} r\left(1-r \sigma^{2} \gamma\right)+1+r \sigma^{2}(1+\gamma)+r^{2} \sigma^{4} \gamma\left(1-\gamma \lambda^{2}\right)} .
\end{aligned}
$$

Equilibrium surplus is

$$
U^{* *}=\frac{1}{2} \frac{2+r \sigma^{2}(1+\gamma-2 \gamma \lambda)+r v^{2}}{v^{2} r\left(1-r \sigma^{2} \gamma\right)+1+r \sigma^{2}(1+\gamma)+r^{2} \sigma^{4} \gamma\left(1-\gamma \lambda^{2}\right)} .
$$

For the same reason as under accounting system $A_{1}$ the principal's equilibrium surplus decreases in $\lambda$.

The basic trade-off between the two accounting systems is that under $A_{1}$ early first- and second-period signals are used, while under $A_{2}$ a more precise signal $y_{1}^{L}$ (as compared to $y_{1}$ ) is used along with a less precise, garbled version of $y_{2}$. For the special case of $\gamma=1$ the trade-off vanishes and $A_{1}$ is always preferred to $A_{2}$. While under $A_{1}$ the principal can use the signals $\left(y_{1}, y_{2}\right)$, under $A_{2}$ she must use the garbled set of performance measures $\left(y_{1}, P_{2}\right)$ to control effort (notice that $y_{1}^{L}=y_{1}$ for $\gamma=1)$. 
For $\gamma<1$, contrasting the principal's surplus attainable in both settings, we obtain Proposition 1 .

Proposition 1 If $\sigma^{2}\left(1+r \sigma^{2}\right)>v^{2}\left(1-r \sigma^{2}\right)$, there exists a critical value $\hat{\gamma} \in(0,1)$ such that $U^{*} \gtreqless U^{* *}$ if $\gamma \gtreqless \hat{\gamma}$. Otherwise, $A_{1}$ strictly dominates $A_{2}$ for all $\gamma \in[0,1]$.

Proposition 1 states that under a certain condition there exists a threshold $\hat{\gamma}$ such that $A_{1}$ will be preferred if $\gamma$ is higher than the threshold. To start off, note that the benefit from reducing noise by using $y_{1}^{L}$ under $A_{2}$ rather than $y_{1}$ under $A_{1}$ is strongest for low values of $\gamma$. Besides, for $\gamma$ low, the covariance effects are insignificant as covariances equal $\operatorname{cov}\left(y_{1}, y_{2}\right)=\sigma^{2} \lambda \gamma$ under $A_{1}$ and $\operatorname{cov}\left(y_{1}^{L}, P_{2}\right)=\sigma^{2} \lambda \gamma$ under $A_{2}$. It follows that, if $A_{2}$ is optimal at all, it must be for low values of $\gamma$.

Comparative statics show that $\hat{\gamma}$ decreases in $v$ (see the proof of Proposition 1). First notice that $v^{2}=\operatorname{Var}(\eta)$ measures the additional noise resulting from using $P_{2}$ under $A_{2}$ as compared to using $y_{2}$ under $A_{1}$. Higher $v$ c.p. reduces the surplus under $A_{2}$ and, therefore, reduces $\hat{\gamma}$. This aspect is reflected in the necessary and sufficient condition for a critical value $\hat{\gamma}$ to exist in Proposition 1. In fact, this condition ensures that for $\gamma=0$, where the benefits from switching from $A_{1}$ to $A_{2}$ are greatest, the surplus under $A_{2}$ is higher than under $A_{1}$. It is fulfilled if $v$ is sufficiently low.

In Fig. 2 we plot the surplus difference $\Delta U=U^{*}-U^{* *}$ as a function of $\gamma$ for a given set of parameters. The critical value is given by $\hat{\gamma}=0.71$.

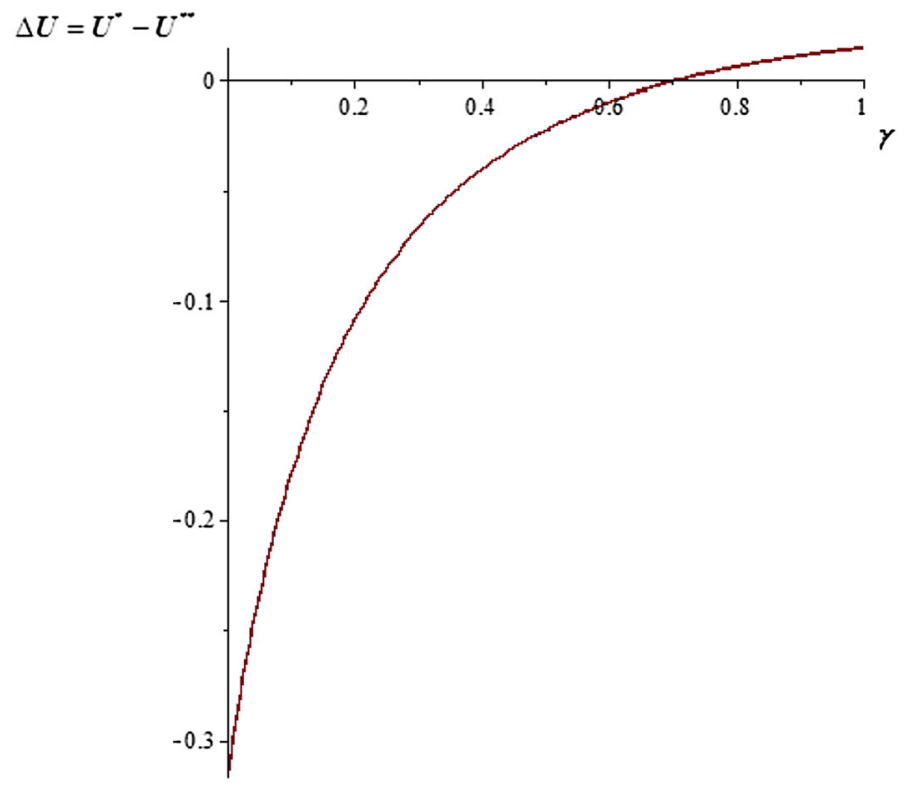

Fig. 2 Optimality of both accounting systems depending on $\gamma$ under full commitment. Parameters of the example: $r=1, \sigma=2, v=5, \lambda=0.9$ 


\section{Limited commitment solutions}

\subsection{Limited commitment, short-term contracts and renegotiation-proofness in a two-period LEN-setting}

In this section we relax our previous assumption that the principal and the agent can commit to a long-term (two-period) incentive contract. We rather assume that parties can only agree on short-term, one-period, contracts. To sustain an equilibrium of the game we follow Christensen et al. (2003). We assume that the principal can commit to a "fair" second-period contract, while the agent can commit to work for two periods for the principal. Note that the equilibrium outcome of a sequence of two short-term contracts, in terms of induced actions and surplus, is identical to the outcome under an optimal renegotiation-proof long-term contract (see Christensen et al. (2003) again). A long-term contract is renegotiation-proof, if the principal has no incentive to change the second-period contract parameters given her information set at $t=1$, i.e., after $y_{1}$ has been observed. We use this equivalence result in our paper. Even though short-term contracts are more descriptive in many real-world settings, working with a renegotiation-proof contract allows to contrast full and limited commitment more easily. Thus, we proceed considering a renegotiation-proof long-term contract in this section.

Christensen et al. (2003) show for a two-period LEN-model that a long-term contract is renegotiation-proof if and only if second-period incentive weights are chosen sequentially optimal. In other words, the optimization problem to be considered under limited commitment is identical to the one from the full commitment setting except that sequentially optimal second-period incentive rates apply.

Importantly, there is a conflict between ex ante efficient and ex post efficient contracting decisions. Ex ante, the principal considers motivating the first- and the second-period effort, holding the variance of the agent's total compensation at bay. At the end of the first period, however, the first-period effort has already been performed and, thus, becomes irrelevant when determining second period contracting parameters. In addition, at the renegotiation stage $y_{1}$ has been observed and, thus, some uncertainty has been resolved. Particularly, at the end of the first period the principal considers solely the variance of the second-period compensation given the information obtained in the first period.

Under full commitment the principal uses all signals for contracting that are (incrementally) informative about the agent's effort. The informativeness principle holds true under limited commitment, too, but under the requirement that signals and their incentive weights have to be chosen sequentially optimal.

In our model, the strength of the sequential rationality (or renegotiationproofness) constraint is driven by two main factors: the correlation of accounting noise $\lambda$ and the fact that under $A_{2}$ both contracting variables must be chosen sequentially optimal, such that there is no possibility to control first-period effort directly. 
In addition to the trade-off between systems identified under full commitment, the effect both accounting systems have on sequentially optimal second-period effort values determines which one is preferred.

\subsection{Early versus late information under limited commitment}

As stated in the previous section, second-period incentive rates are to be chosen sequentially optimal with limited commitment. Thus, in the first step, we determine sequentially optimal incentive rates under both accounting systems. They are chosen to maximize the second-period part of the principal's expected gross output, $a_{2}$, net of its costs and subject to the incentive constraint for second-period effort. Costs cover the agent's disutility of performing effort in period $2, a_{2}^{2} / 2$, and the posterior risk premium to be paid to the agent, $\operatorname{Var}\left(S \mid y_{1}\right)$.

As $P_{2}$ is not informative about $a_{2}$ in the presence of $y_{2}$, under $A_{1} z_{2}=0$ holds, as is the case under full commitment. The sequentially optimal value of $s_{2}$ solves the following problem:

$$
\begin{gathered}
\max _{s_{2}} U_{2}^{A_{1}}=a_{2}-\frac{a_{2}^{2}}{2}-\frac{r}{2} \operatorname{Var}\left(s_{2} y_{2} \mid y_{1}\right) \\
\text { s.t. } a_{2}=s_{2} .
\end{gathered}
$$

For the late accounting information system, $A_{2}$, the coefficients $s$ and $z_{2}$ must be set sequentially optimal. The corresponding program is given by

$$
\begin{aligned}
\max _{s, z_{2}} U_{2}^{A_{2}} & =a_{2}-\frac{a_{2}^{2}}{2}-\frac{r}{2} \operatorname{Var}\left(s y_{1}^{L}+z_{2} P_{2} \mid y_{1}\right) \\
\text { s.t. } a_{2} & =z_{2} .
\end{aligned}
$$

Given that the sequentially optimal incentive weights have been determined, the overall solution under limited commitment will be found by substituting the sequentially optimal incentive rates into the full commitment programs from Sect. 3 and then optimizing for the remaining first period incentive coefficient (under $A_{1}$ ).

Lemma 3 (a) Under $A_{1}$ the optimal renegotiation-proof contract exhibits

$$
\begin{aligned}
& s_{2}^{R *}=\frac{1}{1+r \sigma^{2}\left(1-\gamma^{2} \lambda^{2}\right)}, z_{2}^{R *}=0, \quad \text { and } s_{1}^{R *}=\frac{1+r \sigma^{2}\left(1-\gamma \lambda-\gamma^{2} \lambda^{2}\right)}{\left(1+r \sigma^{2}\right)\left(1+r \sigma^{2}\left(1-\gamma^{2} \lambda^{2}\right)\right)} \\
& \text { The } \quad \text { equilibrium surplus } \quad \text { is } \\
& \frac{1}{2} \frac{r^{2} \sigma^{4}\left(\gamma^{4} \lambda^{4}+2 \gamma^{3} \lambda^{3}-3 \gamma^{2} \lambda^{2}-2 \gamma \lambda+2\right)+2 r \sigma^{2}\left(2\left(1-\gamma^{2} \lambda^{2}\right)-\gamma \lambda\right)+2}{\left(1+r \sigma^{2}\right)\left(1+r \sigma^{2}\left(1-\gamma^{2} \lambda^{2}\right)\right)^{2}}
\end{aligned}
$$

(b) Under $A_{2}$ the optimal renegotiation-proof contract exhibits

$$
s^{R * *}=-\frac{\lambda}{r \sigma^{2}\left(1-\gamma \lambda^{2}\right)+1+r v^{2}}, \quad z_{2}^{R * *}=\frac{1}{r \sigma^{2}\left(1-\gamma \lambda^{2}\right)+1+r v^{2}} .
$$

The equilibrium surplus is given by $U^{R * *}=\frac{1}{2} \frac{1-\lambda^{2}-2 \lambda+r v^{2}(1-2 \lambda)+r \sigma^{2}(1-2 \lambda)\left(1-\gamma \lambda^{2}\right)}{\left(r \sigma^{2}\left(1-\gamma \lambda^{2}\right)+1+r v^{2}\right)^{2}}$. 
Under $A_{1}$ second-period incentive rate $s_{2}$ needs to be chosen sequentially optimal (with $z_{2}=0$ ). Under $A_{2}$, signals $y_{1}^{L}$ and $P_{2}$ are available for contracting only in period two. Accordingly, both incentive rates must be chosen sequentially optimal, i.e., without regarding their incentive effect on the first-period effort. As $y_{1}^{L}$ does not depend on $a_{2}$, it is used purely to hedge some of the risk imposed on the agent by compensating him based on $P_{2}$. At the optimum $s^{R * *}=-z_{2}^{R * *} \lambda$ holds. $z_{2}^{R * *}$ is positive to motivate effort and $s^{R * *}>(=)<0$ if $\lambda<(=)>0$ applies. Using an incentive rate with the opposite sign of the correlation coefficient triggers the hedge effect.

The sign of the correlation coefficient $\lambda$ also determines whether induced secondperiod effort under both systems is too high or too low compared to its optimal full commitment value.

Lemma 4 For $\gamma \in(0,1)$ : Under both accounting systems sequentially optimal second-period effort is too low (high) compared to the full commitment value if $\lambda<(>) 0$.

Recall from the analysis of the full commitment setting that positively correlated noise ceteris paribus leads to low optimal effort incentives, while negative correlation results in an intertemporal insurance effect such that high effort incentives will be set. Ex post, that is after the first-period early signal $y_{1}$ has been observed, however, only the posterior variance matters. As there are no intertemporal insurance opportunities anymore, the sign of the correlation coefficient becomes irrelevant. As a consequence, the ex post optimal value of $a_{2}$ is too high whenever correlation is positive and too low whenever correlation is negative from an ex ante perspective. Limited commitment, therefore, results in distorted incentives and reduces expected payoffs of the principal whenever periods are correlated. This holds true for both accounting systems.

Having compared limited commitment to full commitment we continue juxtaposing the two accounting systems under limited commitment starting with Proposition 2.

$$
\text { Proposition } 2 \text { (a) For } \lambda=0 \quad U^{R *}>U^{R * *} \text {. (b) Assume } \lambda<0 \text {. If }
$$
$-\left(1+r \sigma^{2}\right)\left((1+\lambda)^{2}+r \sigma^{2}(1+2 \lambda)\right)>r v^{2}\left[2 r v^{2}+(3+2 \lambda)\left(1+r \sigma^{2}\right)\right]$, there exists a critical value $\hat{\gamma}^{R} \in(0,1)$ such that $U^{R *} \gtreqless U^{R * *}$ if $\gamma_{<} \hat{\gamma}^{R}$. Otherwise, $A_{1}$ strictly dominates $A_{2}$ for all $\gamma \in(0,1)$.

Consider part (a) of Proposition 2. If correlation is absent, two independent periods are present under $A_{1}$. First-period effort is controlled by $y_{1}$ and secondperiod effort by $y_{2}$. Under $A_{2}$, in contrast, no correlation implies $s^{R *}=0$ as no hedging opportunity exists. Thus, the principal is left with a single measure $P_{2}$ to control $a_{2}$. First period effort cannot be motivated at all. Moreover, $P_{2}$ being a garbling of $y_{2}$ is an inferior performance measure. It follows that $A_{1}$ is the preferred accounting system.

For $\lambda<0$ (part b) we obtain a result closely related to that from Proposition 1: If a certain condition is fulfilled, there exists a critical value $\hat{\gamma}^{R}$ such that $A_{1}\left(A_{2}\right)$ is the preferred system if $\gamma>(<) \hat{\gamma}^{R}$. Similar to Proposition 1 , this condition ensures that 
the surplus under $A_{2}$ is higher if the maximal reduction in variance is present $(\gamma=0)$. For this to be true, once again $v$ needs to be sufficiently low. In addition, a sufficiently small $\lambda(<-0.5)$ is a necessary condition. The intuition is slightly different from the full commitment one. Recall that under full commitment the comparative advantage of system $A_{2}$ is that the principal can use a less noisy late first-period signal to control the first-period action. Under limited commitment, in contrast, $y_{1}^{L}$ will be used in the ex post optimal contract as it is informative about $a_{2}$ given $P_{2}$. Thus, $a_{1}$ cannot be directly controlled under late accounting. However, under some conditions the ex post optimal use of $y_{1}^{L}$ does not heavily deviate from the ex ante optimal use. A prerequisite for this to be true is that $\lambda$ is sufficiently negative. To see this, notice that the agent chooses $a_{1}^{R * *}=s^{R * *}=-z_{2}^{R * *} \lambda$. Only for sufficiently negatively correlated noise the induced first-period effort is significantly positive. Thus, only in this case a low variance of the first-period late accounting signal (low $\gamma$ ) is valuable, as it allows to induce a positive first-period effort at comparatively low cost. Additionally, a low $\operatorname{Var}(\eta)=v^{2}$ implies that the cost of using $P_{2}=y_{2}+\eta$ rather than $y_{2}$ is small. If both effects are simultaneously present, incentives with respect to $a_{1}$ are not heavily distorted and $P_{2}$ is a sufficiently good performance measure. As a result $A_{2}$ is preferred to $A_{1}$.

If noise is positively correlated over time, we obtain a qualitatively different result, as is demonstrated in Proposition 3.

Proposition 3 If $\lambda>0$ and sufficiently high and $v$ sufficiently high, there exists $a$ critical value $\hat{\gamma}^{R} \in(0,1)$ such that $U^{R *}>U^{R * *}$ if $\gamma \gtreqless \hat{\gamma}^{R}$ for $\gamma \in(0,1)$.

Notice that this result, in some sense, reverses the results from Proposition 1 and 2. While our previous results showed that the late accounting system becomes more favorable the stronger the reduction in noise over time, that is the lower $\gamma$, now the opposite holds true. As presented in Proposition 3, $A_{2}$ is possibly preferred over $A_{1}$ if noise persists, that is for high values of $\gamma$. This becomes immediately apparent from the numerical example depicted in Fig. 3. We use identical parameter values as in Fig. 2 (full commitment), but obtain reversed optimality regions for the two accounting systems. For $\gamma<\hat{\gamma}^{R}=0.91$ the early accounting system is optimal and for $\gamma>0.91$ the late accounting system.

To get the intuition consider first the extreme cases of $\gamma \rightarrow 0$ and $\gamma \rightarrow 1$. $^{11}$

Under $A_{1}, \gamma \rightarrow 0$ has similar effects as $\lambda=0 . \gamma \rightarrow 0$ implies that $\varepsilon_{t} s$ are (quasi) deterministic and thus all noise related to $y_{1}$ and $y_{2}$ is uncorrelated. Thus, the principal is left with two independent periods, controlled by $y_{1}$ and $y_{2}$, respectively. This renders the sequential optimality requirement obsolete in equilibrium and, therefore, full commitment results are obtained. Under $A_{2}$ the correlation effect is negligible as well, but nonetheless $s$ will be used to hedge some risk related to $P_{2}$ even if the effect is only marginal. Importantly, the choice of $s$ affects first-period

\footnotetext{
${ }^{11}$ For $\gamma=0$ and $\gamma=1$ any value of $s$ is sequentially optimal under $A_{2}$. In the first case $y_{1}^{L}$ turns out to be deterministic, in the second case $y_{1}^{L}=y_{1}$ holds. Thus, these are special cases where $y_{1}^{L}$ can be ex ante optimally contracted upon under $A_{2}$. To avoid degenerated results, we consider $\gamma \rightarrow 0$ and $\gamma \rightarrow 1$ which means that we consider $\gamma=0$ and $\gamma=1$ except for $s^{R * *}$ for which we consider the limits for $\gamma \rightarrow 0$ and $\gamma \rightarrow 1$ such that there is an ex post optimal value for $s^{R * *}$.
} 


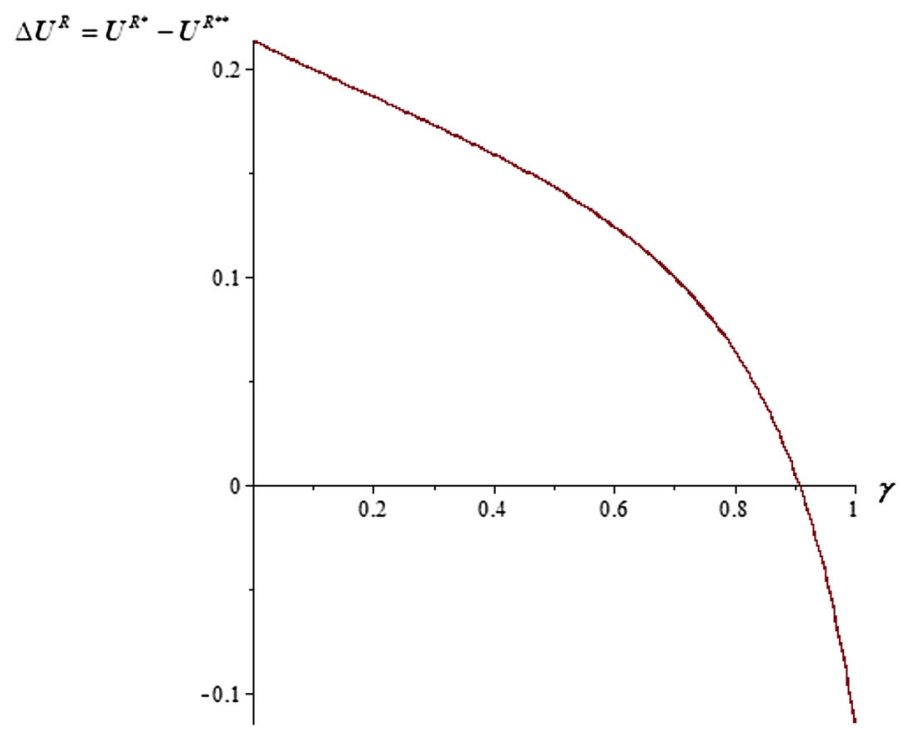

Fig. 3 Optimality of both accounting systems depending on $\gamma$ under limited commitment. Parameters of the example: $r=1, \sigma=2, v=5, \lambda=0.9$

effort. In particular, for positively correlated noise $s^{R * *}$ is negative, inducing a negative first-period effort. Contrasting $A_{1}$ and $A_{2}$ now shows that $A_{1}$ allows to induce positive first-period effort while $A_{2}$ does not. Moreover, with $A_{1} y_{2}$ is available to control second-period effort, while only its garbling $P_{2}$ can be used under $A_{2}$. Both aspects work in favor of $A_{1}$. Consequently, $A_{2}$ is dominated by $A_{1}$ for $\gamma \rightarrow 0$.

Now consider the other extreme $\gamma \rightarrow 1$. Under full commitment it was shown that $A_{2}$ is strictly dominated by $A_{1}$. This results from the fact that under $A_{2}$ noise reduction from the early to the late signal is absent and the "garbling" $P_{2}$ must be used rather than $y_{2}$. Any comparative advantage of late accounting, therefore, vanishes for $\gamma \rightarrow 1$. However, under limited commitment the very disadvantage of $A_{2}$ can turn into an advantage.

Under $A_{1}$ with $\gamma \rightarrow 1$ the principal uses $y_{1}$ and $y_{2}$ for contracting where $y_{2}$ must be used sequentially optimal. Under $A_{2}$ the principal uses $y_{1}^{L}=y_{1}$ and $P_{2}$ for contracting, both being used sequentially optimal. As $P_{2}$ is a garbling of $y_{2}$, three facts immediately follow: First, ceteris paribus the risk premium to be paid to the agent is lower under $A_{1}$, both ex ante and ex post. Second, incentives for $a_{2}$ are higher powered under $A_{1}$. Third, under $A_{2}$ no positive first-period effort can be induced as $s^{R * *}<0$. First and third aspects clearly favor system $A_{1}$.

The effect of higher powered second-period effort under $A_{1}$ relative to $A_{2}$, however, is not clear. For positively correlated accounting noise, too high powered second-period incentives are set under $A_{1}$ compared to the full commitment setting. This holds true for $A_{2}$, too. Importantly though, second-period equilibrium incentives are lower under $A_{2}$ than under $A_{1}$ due to the higher ex post risk 
premium related to the garbled performance measure. Moving from the early to the late accounting system thus may reduce distortions from the ex ante efficient second-period effort. Of course, this effect only works if the incentives under $A_{2}$ turn out to be not too low. If this effect outweighs the additional risk premium and the loss from dysfunctional first-period working incentives, $A_{2}$ is even preferred to $A_{1}$ given $\gamma \rightarrow 1$. Hence, at least under some circumstances there exists a critical value for $\gamma$ such that the late accounting system becomes optimal, if $\gamma$ exceeds the critical value.

A necessary condition for this result to apply is a strong positive correlation between $y_{1}$ and $y_{2}$. Intuitively the higher the (positive) $\lambda$ the stronger the distortion of the second-period sequentially optimal effort from the ex ante optimal value under $A_{1}$. Thus, in this case the benefits from avoiding high powered incentives are the greatest.

Finally, the additional noise in $P_{2}$, measured by $v$, turns out to be critical for the reversed result to exist. A high value of $v$ has two effects under $A_{2}$ : First, secondperiod effort is low and so is absolute value of $s^{R * *}$. The first effect implies that second-period effort under $A_{2}$ is much lower than under $A_{1}$. This favors $A_{2}$ as due to the high correlation second-period incentives under $A_{1}$ are far too strong. The effect of high $v$ on first-period effort under $A_{2}$ is also positive because low absolute value of $s^{R * *}$ keeps dysfunctional first-period working incentives at bay.

\section{Extensions}

As discussed in some detail in Sects. 1 and 2, we assume in our analysis that the late second-period accounting measure $y_{2}^{L}$ is unavailable for contracting. At the same time we presume that some non-accounting measure is present in period two to allow for second-period effort control at all. Both assumptions are not only critical for our results but also might appear somewhat ad hoc. In order to allow readers to comprehend the consequences of these specific modeling choices we discuss effects of what we consider straightforward alternative modeling assumptions below.

First, let us assume that only the accounting measures are available (no $P_{2}$ ), but that late reporting does not restrict the availability for contracting. In our two-period setting, this would imply $y_{2}^{L}$ could be used for contracting once it is reported in period three. To be consistent, under limited commitment an incentive rate on $y_{2}^{L}$ would be chosen sequentially optimal at $t=2$. With full commitment, obviously the late system dominates the early one as more precise contracting information can be used to control both efforts. No trade-off is present any more. With limited commitment, however, it will be impossible to motivate any effort at all. To see that, note that $a_{2}$ has already been performed in period two. As the agent is risk averse it is optimal for the principal to put zero weight on a performance measure $y_{2}^{L}$ at $t=2$ if it is not completely noiseless. With respect to the first-period effort the same rationale applies. Recall that the incentive rate on $y_{1}^{L}$ was chosen to optimally hedge compensation risk related to the use of $P_{2}$. In the absence of such a measure it is ex post optimal to set $s=0$ such that no effort is motivated at all. To sum up, late 
reporting dominates early reporting with full commitment, and early reporting dominates late reporting with limited commitment. This result, again, holds if the timing of reporting is irrelevant for contractability and no performance measures besides accounting measures can be used. We found both assumptions not very convincing and thus decided against them.

Second, we could have assumed that contracting on $y_{2}^{L}$ is impossible without allowing for an alternative measure $P_{2}$. This implies that under full commitment it is impossible to motivate any effort in the second period under the late system which works in favor of the early one. Under limited commitment the results resemble the ones from above. Due to the absence of any performance measure the principal is unable to motivate second-period effort under $A_{2}$. Due to the ex post optimal choice of the incentive rate on $y_{1}^{L}$ she would set $s=0$, again implying no first-period effort motivation. $A_{1}$ would dominate $A_{2}$. Once more, the implausible assumption of using accounting measures only kept us from using this setting.

Finally, if we consider availability of a non-accounting measure in period two a reasonable assumption, it would be natural to allow for such a measure in period one as well. We abstained from doing so only for tractability reasons. Including some $P_{1}$ measure would not affect our solutions under full commitment. In fact being a garbling of $y_{1}$ and $y_{1}^{L}$ it would not be used in an optimal contract. With limited commitment the additional measure would only be used under the late system. It would help to control the first-period effort since it could be chosen optimally ex ante. This would work in favor of the late system as opposed to the early one, but would not change our findings qualitatively. It would also render all our results messier than necessary, which is why we opted against it.

\section{Conclusion}

In this paper we contrast early versus late reporting of accounting information in a two-period agency setting. Two accounting systems are present and both produce identical information that is correlated over time. However, the reporting dates of the signals produced differ due to a different emphasis on relevance and reliability, immanent in the set of standards applied. This reflects that for some items reported in financial statements-certainly not for every single one-a trade-off between relevance and reliability exists. Ready examples besides traditional revenue recognition, as stated in the introductory example, are fair value recognition for assets or capitalization of self-created intangibles.

If relevance is emphasized, the signal is reported in the period in which it is produced. With emphasis on reliability, reporting is delayed by one period at the benefit of reducing the noise inherent in the signal. Reporting, however, is a necessary condition for contractability in our setting. Moreover, we build on the fact that the farther in the distant future a measure is reported, the less useful it becomes for contracting. We reflect this idea in our model by restricting contractability to measures reported throughout the two-period horizon of our game. As a result, late reporting prevents the second-period accounting signal from being directly available 
for contracting. To allow for motivation of the second-period effort at all, we assume that some, possibly distorted, non-accounting signal is present to be used as a performance measure.

Within this structure we derive optimal compensation contracts in a full commitment setting as opposed to a limited commitment setting.

With full commitment the comparative advantage of the early information system is availability of a second-period accounting measure for contracting. The late system relatively benefits from the less noisy first-period measure that is available for contracting. Which system is preferred overall depends on which effect dominates the other.

With limited commitment our results differ depending on the type of intertemporal correlation over time. If negative correlation is present, the early system dominates the late system if the non-accounting performance measure is too much of a garbling of the second-period accounting measure. If the garbling is moderate, the early system dominates the late one only if the reduction in noise over time is below a critical value. Otherwise the late system is preferred. Thus with negative intertemporal correlation our results qualitatively resemble the full commitment ones.

With positive intertemporal correlation this is no longer the case. Rather, we find that in some settings the early system is preferred if the reduction in noise from the early to the late signal is large, while the late system dominates if noise reduction is small.

The result stems from the fact that positive correlation creates excessively high second-period incentives under both accounting systems. With the late system in place, however, this effect is weaker as a distorted non-accounting measure is used less heavily in equilibrium. If this favorable effect more than outweighs the unfavorable risk and first-period effort effects related to the late system, it dominates the early one.

Summing up, our results show that a trade-off is present between accounting systems emphasizing relevance and reliability, implying that there is no strong point for either favoring early or late, or alternatively more or less reliable or relevant, reporting from a contracting perspective. This insight is amplified by the finding that the identified trade-off is sensitive to the commitment assumption present and the type of intertemporal correlation.

Focusing once more on limited commitment as the probably most relevant setting, empirical implications from our study can be summarized as follows: We would expect to see timely accounting (emphasis on relevance) if negative correlation is present and the reduction in noise over time is not too strong. An example for such a setting could be the use of revenue for performance measurement in a specialty goods industry as described in the introductory example. In contrast, if noise reduction is severe, an emphasis on reliability is likely, in particular, if an appropriate non accounting measure is available for contracting as well. With positive correlation in accounting signals the results reverse: Emphasis on relevance is likely to be observed if noise reduction is strong and a precise non-accounting measure is available. 
Open Access This article is distributed under the terms of the Creative Commons Attribution 4.0 International License (http://creativecommons.org/licenses/by/4.0/), which permits unrestricted use, distribution, and reproduction in any medium, provided you give appropriate credit to the original author(s) and the source, provide a link to the Creative Commons license, and indicate if changes were made.

\section{Appendix}

Proof of Lemma 1 With the agent's participation constraint binding the principal's problem under $A_{1}$ is given by

$$
\begin{aligned}
& \max _{s_{1}, s_{2}, z_{2}} U^{A_{1}}=a_{1}+a_{2}-\frac{a_{1}^{2}}{2}-\frac{a_{2}^{2}}{2}-\frac{r}{2} \operatorname{Var}\left(s_{1} y_{1}+s_{2} y_{2}+z_{2} P_{2}\right) \\
& \text { subject to } \\
& a_{1}=s_{1}, \\
& a_{2}=s_{2}+z_{2} .
\end{aligned}
$$

The variance is given by $\operatorname{Var}\left(s_{1} y_{1}+s_{2} y_{2}+z_{2} P_{2}\right)=\operatorname{Var}\left(s_{1} y_{1}+\left(s_{2}+z_{2}\right) y_{2}+z_{2} \eta\right)$.

To avoid $\operatorname{Var}\left(z_{2} \eta\right)$ it is always optimal to set $z_{2}=0$.

The remaining variance is given by $\operatorname{Var}\left(s_{1} y_{1}+s_{2} y_{2}\right)=\sigma^{2}\left[s_{1}^{2}+s_{2}^{2}+2 s_{1} s_{2} \gamma \lambda\right]$. By inserting the agent's action choices into $U^{A_{1}}$ the principal's problem becomes

$$
\max _{s_{1}, s_{2}} U^{A_{1}}=s_{1}+s_{2}-\frac{s_{1}^{2}}{2}-\frac{s_{2}^{2}}{2}-\frac{r}{2} \sigma^{2}\left[s_{1}^{2}+s_{2}^{2}+2 s_{1} s_{2} \gamma \lambda\right] .
$$

The first-order conditions for the principal's problem are:

$$
\begin{aligned}
& \frac{\partial U^{A_{1}}}{\partial s_{1}}=1-s_{1}-r \sigma^{2}\left(\gamma \lambda s_{2}+s_{1}\right)=0 \\
& \frac{\partial U^{A_{1}}}{\partial s_{2}}=1-s_{2}-r \sigma^{2}\left(\gamma \lambda s_{1}+s_{2}\right)=0
\end{aligned}
$$

Solving for $\left(s_{1}, s_{2}\right)$ we obtain

$$
s_{1}^{*}=s_{2}^{*}=\frac{1}{1+r \sigma^{2}(1+\gamma \lambda)} .
$$

Equilibrium surplus is

$$
U^{*}=U^{A_{1}}\left(s_{1}^{*}, s_{2}^{*}\right)=\frac{1}{1+r \sigma^{2}(1+\gamma \lambda)} .
$$

Proof of Lemma 2 Similar to the proof of Lemma 1 the principal's objective function under $A_{2}$ is given by 


$$
\begin{gathered}
\max _{s, z_{2}} U^{A_{2}}=a_{1}+a_{2}- \\
-\frac{a_{1}^{2}}{2}-\frac{a_{2}^{2}}{2}-\frac{r}{2} \operatorname{Var}\left(s y_{1}^{L}+z_{2} P_{2}\right) \\
\text { subject to } \\
a_{1}=s \\
a_{2}=z_{2} .
\end{gathered}
$$

The variance is given by $\operatorname{Var}\left(s y_{1}^{L}+z_{2} P_{2}\right)=\operatorname{Var}\left(s y_{1}^{L}+z_{2} y_{2}+z_{2} \eta\right)=$ $\sigma^{2}\left[s^{2} \gamma+z_{2}^{2}+2 \gamma \lambda s z_{2}\right]+z_{2}^{2} v^{2}$.

By inserting the agent's action choices into $U^{A_{2}}$ the principal's problem becomes

$$
\max _{s, z_{2}} U^{A_{2}}=s+z_{2}-\frac{s^{2}}{2}-\frac{z_{2}^{2}}{2}-\frac{r}{2}\left(\sigma^{2}\left[s^{2} \gamma+z_{2}^{2}+2 \gamma \lambda s z_{2}\right]+z_{2}^{2} v^{2}\right)
$$

The first-order conditions are

$$
\begin{gathered}
\frac{\partial U^{A_{2}}}{\partial s}=1-s-r \sigma^{2}\left(\gamma \lambda z_{2}+\gamma s\right)=0 \\
\frac{\partial U^{A_{2}}}{\partial z_{2}}=1-z_{2}-r\left(\sigma^{2}\left[\gamma \lambda s+z_{2}\right]+z_{2} v^{2}\right)=0 .
\end{gathered}
$$

Solving the first-order conditions for $\left(s, z_{2}\right)$ yields:

$$
\begin{aligned}
s^{* *} & =\frac{1+r \sigma^{2}(1-\gamma \lambda)+r v^{2}}{v^{2} r\left(1-r \sigma^{2} \gamma\right)+1+r \sigma^{2}(1+\gamma)+r^{2} \sigma^{4} \gamma\left(1-\gamma \lambda^{2}\right)}, \\
z_{2}^{* *} & =\frac{1+r \sigma^{2} \gamma(1-\lambda)}{v^{2} r\left(1-r \sigma^{2} \gamma\right)+1+r \sigma^{2}(1+\gamma)+r^{2} \sigma^{4} \gamma\left(1-\gamma \lambda^{2}\right)} .
\end{aligned}
$$

Equilibrium surplus is

$$
U^{* *}=U^{A_{2}}\left(s^{* *}, z_{2}^{* *}\right)=\frac{1}{2} \frac{2+r \sigma^{2}(1+\gamma-2 \gamma \lambda)+r v^{2}}{v^{2} r\left(1-r \sigma^{2} \gamma\right)+1+r \sigma^{2}(1+\gamma)+r^{2} \sigma^{4} \gamma\left(1-\gamma \lambda^{2}\right)} .
$$

Proof of Proposition 1 The difference in surpluses is given by $\Delta U=U^{*}-U^{* *}=\frac{1}{2} r \frac{(1-\gamma) \sigma^{2}\left(1+r \sigma^{2}(1-\gamma \lambda)\right)+v^{2}\left(r \sigma^{2}(\gamma \lambda-2 \gamma+1)-1\right)}{\left(1+r \sigma^{2}(1+\gamma \lambda)\right)\left(r \sigma^{2}\left(r \sigma^{2} \gamma\left(\gamma \lambda^{2}-1\right)-1-\gamma\right)-1-r v^{2}\left(1+r \sigma^{2} \gamma\right)\right)}$, where $U^{*}$ and $U^{* *}$ are defined in Lemma 1 and Lemma 2, respectively. If we solve $\Delta U=0$ for $\gamma$ we obtain two solutions: $\gamma_{1}=\frac{1}{2} \frac{1}{\lambda r \sigma^{2}}(H+\sqrt{M})$ and $\gamma_{2}=-\frac{1}{2} \frac{1}{\lambda r \sigma^{2}}(-H+\sqrt{M})$ with

$$
\begin{gathered}
H=\left(1+r \sigma^{2}(1+\lambda)\right)+r v^{2}(2-\lambda)>0 \\
M=\left(r \sigma^{2}(\lambda-1)-1\right)^{2}-2 r v^{2}(2+\lambda)\left(r \sigma^{2}(\lambda-1)-1\right)+r^{2} v^{4}(\lambda-2)^{2} .
\end{gathered}
$$

Notice that $H>2 \lambda r \sigma^{2}$. Thus, $\gamma_{1}$ is either negative or higher than 1 . It follows that if there is a threshold $\hat{\gamma} \in(0,1)$ such that $\Delta U(\hat{\gamma})=0$ then $\hat{\gamma}=\gamma_{2}$. 
Notice that $\Delta U(\gamma=1)>0$. The reason is that for $\gamma=1 y_{1}^{L}=y_{1}$ holds such that in equilibrium under $A_{1}$ the principal contracts on $y_{1}$ and $y_{2}$ while under $A_{2}$ she contracts on $y_{1}$ and $P_{2}, P_{2}$ being a garbling of $y_{2}$. Furthermore,

$$
\Delta U(\gamma=0)=\frac{1}{2} \frac{r\left[v^{2}\left(r \sigma^{2}-1\right)+\sigma^{2}\left(1+r \sigma^{2}\right)\right]}{\left(1+r \sigma^{2}\right)\left(-\left(1+r \sigma^{2}\right)-r v^{2}\right)} .
$$

The denominator of $\Delta U(\gamma=0)$ is negative. Thus, if $v^{2}\left(r \sigma^{2}-1\right)+\sigma^{2}\left(1+r \sigma^{2}\right)>0$ $\Delta U(\gamma=0)<0$ and due to the intermediate value theorem then there exists a critical value $\hat{\gamma}$ such that $\Delta U(\hat{\gamma})=0$. This critical value is unique and must be equal to $\gamma_{2}$. If $\Delta U(\gamma=0)>0$ then if $A_{2}$ would be optimal for some values of $\gamma$ there must be at least two critical values for $\gamma$ from the set $(0,1)$ where $\Delta U(\gamma)=0$ holds true. However, as we know that there is at most one critical value from this set $\left(\gamma_{2}\right)$, if $\Delta U(\gamma=0)>0, \Delta U>0$ for all $\gamma \in[0,1]$ must hold true.

Comparative statics: The marginal effect of increasing $v$ on $\Delta U$ is given by

$$
\frac{\partial \Delta U}{\partial \nu}=\frac{r v\left(r \sigma^{2} \gamma(\lambda-1)-1\right)^{2}}{\left(\left[r^{2} \sigma^{4} \gamma\left(\gamma \lambda^{2}-1\right)-r \sigma^{2}(1+\gamma)-1\right]-r v^{2}\left(1+r \sigma^{2} \gamma\right)\right)^{2}}>0 .
$$

Hence, if the critical value $\hat{\gamma}=\gamma_{2}$ exists, it is decreasing in $v$.

Proof of Lemma 3 a) Under $A_{1}$ the renegotiation-proof second-period incentive rates are the solution to the following problem

$$
\begin{aligned}
& \max _{s_{2}, z_{2}} U_{2}^{A_{1}}=a_{2}-\frac{a_{2}^{2}}{2}-\frac{r}{2} \operatorname{Var}\left(s_{2} y_{2}+z_{2} P_{2} \mid y_{1}\right) \\
& \text { s.t. } a_{2}=s_{2}+z_{2} .
\end{aligned}
$$

Like under full commitment as $P_{2}$ is a garbling of $y_{2}, z_{2}=0$ results. Thus, the posterior variance reduces to $\operatorname{Var}\left(s_{2} y_{2} \mid y_{1}\right)$ with $\operatorname{Var}\left(y_{2} \mid y_{1}\right)=\operatorname{Var}\left(y_{2}\right)-\frac{\operatorname{Cov}\left(y_{2}, y_{1}\right)^{2}}{\operatorname{Var}\left(y_{1}\right)}=$ $\sigma^{2}-\frac{\left(\sigma^{2} \gamma \lambda\right)^{2}}{\sigma^{2}}=\sigma^{2} \cdot\left[1-\lambda^{2} \gamma^{2}\right]$ [see DeGroot (1970), p. 55, formula (17)]. By inserting $a_{2}=s_{2}$ into $U_{2}^{A_{1}}$ the principal's problem becomes

$$
\max _{s_{2}} U_{2}^{A_{1}}=s_{2}-\frac{s_{2}^{2}}{2}-\frac{r}{2} s_{2}^{2} \sigma^{2} \cdot\left[1-\lambda^{2} \gamma^{2}\right] .
$$

From the first-order condition

$$
\frac{\partial U_{2}^{A_{1}}}{\partial s_{2}}=1-s_{2}-s_{2} r \sigma^{2}\left(1-\lambda^{2} \gamma^{2}\right)=0
$$

we obtain $s_{2}^{R *}=\frac{1}{\left.1+r \sigma^{2}\left(1-\gamma^{2} \lambda^{2}\right)\right)}$.

To solve for the equilibrium of the overall game we optimize the principal's objective function $U^{A_{1}}$ (given in Lemma 1) over $s_{1}$ given $s_{2}=s_{2}^{R *}$ :

$$
\max _{s_{1}} U^{A_{1}}=s_{1}+s_{2}^{R *}-\frac{s_{1}^{2}}{2}-\frac{\left(s_{2}^{R *}\right)^{2}}{2}-\frac{r}{2} \sigma^{2}\left[s_{1}^{2}+\left(s_{2}^{R *}\right)^{2}+2 s_{1}\left(s_{2}^{R *}\right)^{2} \gamma \lambda\right] .
$$


From the first-order condition $\frac{\partial U^{A_{1}}}{\partial s_{1}}=1-s_{1}-r \sigma^{2}\left(\gamma \lambda s_{2}^{R *}+s_{1}\right)=0$ we obtain $s_{1}^{R *}=$ $\frac{1+r \sigma^{2}\left(1-\gamma \lambda-\gamma^{2} \lambda^{2}\right)}{\left(1+r \sigma^{2}\right)\left(1+r \sigma^{2}\left(1-\gamma^{2} \lambda^{2}\right)\right)}$. The equilibrium surplus is

$$
\begin{aligned}
U^{R *} & =U^{A_{1}}\left(s_{1}^{R *}, s_{2}^{R *}\right) \\
& =\frac{1}{2} \frac{r^{2} \sigma^{4}\left(\gamma^{4} \lambda^{4}+2 \gamma^{3} \lambda^{3}-3 \gamma^{2} \lambda^{2}-2 \gamma \lambda+2\right)+2 r \sigma^{2}\left(2\left(1-\gamma^{2} \lambda^{2}\right)-\gamma \lambda\right)+2}{\left(1+r \sigma^{2}\right)\left(1+r \sigma^{2}\left(1-\gamma^{2} \lambda^{2}\right)\right)^{2}} .
\end{aligned}
$$

b) Under $A_{2}$ the principal's problem to determine the renegotiation-proof secondperiod incentive rates is given by

$$
\begin{aligned}
& \max _{s, z_{2}} U_{2}^{A_{2}}=a_{2}-\frac{a_{2}^{2}}{2}-\frac{r}{2} \operatorname{Var}\left(s y_{1}^{L}+z_{2} P_{2} \mid y_{1}\right) \\
& \text { s.t. } a_{2}=z_{2} .
\end{aligned}
$$

Similar to part a), with $X_{2}=s y_{1}^{L}+z_{2} P_{2}$ the posterior variance $\operatorname{Var}\left(X_{2} \mid y_{1}\right)$ can be calculated as

$$
\begin{aligned}
\operatorname{Var}\left(X_{2} \mid y_{1}\right)= & \operatorname{Var}\left(X_{2}\right)-\frac{\operatorname{Cov}\left(X_{2}, y_{1}\right)^{2}}{\operatorname{Var}\left(y_{1}\right)}=\sigma^{2}\left(s^{2} \gamma+z_{2}^{2}+2 s z_{2} \gamma \lambda\right) \\
& +z_{2}^{2} v^{2}-\frac{\left(\sigma^{2} \gamma\left(z_{2} \lambda+s\right)\right)^{2}}{\sigma^{2}} \\
= & \sigma^{2} \cdot\left[s^{2} \gamma(1-\gamma)+z_{2}^{2}\left(1-\gamma^{2} \lambda^{2}\right)+2 s z_{2} \gamma(1-\gamma) \lambda\right]+z_{2}^{2} v^{2}
\end{aligned}
$$

By inserting $a_{2}=z_{2}$ into $U_{2}^{A_{2}}$ the principal's problem becomes

$$
\max _{s, z_{2}} U_{2}^{A_{2}}=z_{2}-\frac{z_{2}^{2}}{2}-\frac{r}{2}\left(\sigma^{2} \cdot\left[s^{2} \gamma(1-\gamma)+z_{2}^{2}\left(1-\gamma^{2} \lambda^{2}\right)+2 s z_{2} \gamma(1-\gamma) \lambda\right]+z_{2}^{2} v^{2}\right) \text {. }
$$

From the first-order conditions

$$
\begin{aligned}
& \frac{\partial U_{2}^{A_{2}}}{\partial s}=-r \sigma^{2} \gamma(1-\gamma)\left(s+z_{2} \lambda\right)=0 \\
& \frac{\partial U_{2}^{A_{2}}}{\partial z_{2}}=1-z_{2}-r\left[\sigma^{2}\left(z_{2}\left(1-\gamma^{2} \lambda^{2}\right)+(1-\gamma) \gamma(s \lambda)\right)+z_{2} v^{2}\right]=0
\end{aligned}
$$

we obtain $s^{R * *}=-\frac{\lambda}{r \sigma^{2}\left(1-\gamma \lambda^{2}\right)+1+r v^{2}}$ and $z_{2}^{R * *}=\frac{1}{r \sigma^{2}\left(1-\gamma \lambda^{2}\right)+1+r \nu^{2}}$.

The equilibrium surplus is given by

$$
U^{R * *}=U^{A_{2}}\left(s^{R * *}, z_{2}^{R * *}\right)=\frac{1}{2} \frac{1-\lambda^{2}-2 \lambda+r v^{2}(1-2 \lambda)+r \sigma^{2}(1-2 \lambda)\left(1-\gamma \lambda^{2}\right)}{\left(r \sigma^{2}\left(1-\gamma \lambda^{2}\right)+1+r v^{2}\right)^{2}} .
$$

Proof of Lemma 4 Under $A_{1}$ the agent chooses $a_{2}=s_{2}$. Thus, under $A_{1}$ the difference between induced effort in period 2 under full and limited commitment is 
given by $\Delta a_{2}^{*}=a_{2}^{*}-a_{2}^{R *}=s_{2}^{*}-s_{2}^{R *}$ where $s_{2}^{*}$ and $s_{2}^{R *}$ are given in Lemmas 1 and 3a), respectively. We obtain $\Delta a_{2}^{*}=\frac{\lambda \gamma r \sigma^{2}(1+\gamma \lambda)}{\left(-1-r \sigma^{2}\left(1-\gamma \lambda^{2}\right)\right)\left(1+r \sigma^{2}\left(1-\gamma^{2} \lambda^{2}\right)\right)}<0$ if $\lambda \leq 0$.

Under $A_{2}$ the agent chooses $a_{2}=z_{2}$. Thus, $\Delta a_{2}^{* *}=z_{2}^{* *}-z_{2}^{R * *}$ where $z_{2}^{* *}$ and $z_{2}^{R * *}$ are given in Lemma 2 and Lemma 3b), respectively. We obtain $\Delta a_{2}^{* *}=$ $\frac{\lambda \gamma r \sigma^{2}\left(r \sigma^{2}\left(\gamma \lambda^{2}-1\right)-1-\lambda-r v^{2}\right)}{\left(r \sigma^{2}\left(\gamma \lambda^{2}-1\right)-1-r v^{2}\right)\left(r^{2} \sigma^{4} \gamma\left(\gamma \lambda^{2}-1\right)-r \sigma^{2}(1+\gamma)-1-r v^{2}\left(1+\gamma r \sigma^{2}\right)\right)}<0$ if $\lambda \leq 0$.

Proof of Proposition 2 Define $\Delta U^{R}=U^{R *}-U^{R * *}$, where $U^{R *}$ and $U^{R * *}$ are given in Lemma 3.

a): For $\lambda=0 \Delta U^{R}=\frac{1}{2} \frac{1+r \sigma^{2}+2 r v^{2}}{\left(1+r \sigma^{2}\right)\left(1+r \sigma^{2}+r v^{2}\right)}>0$ results.

b): Assume $\lambda<0$. We calculate ${ }^{12}$

$\Delta U^{R}(\gamma \rightarrow 0)=\frac{1}{2} \frac{\left(1+r \sigma^{2}\right)\left(r \sigma^{2}(1+2 \lambda)+(1+\lambda)^{2}\right)+r v^{2}\left[2 r v^{2}+\left(1+r \sigma^{2}\right)(3+2 \lambda)\right]}{\left(1+r \sigma^{2}\right)\left(1+r \sigma^{2}+r v^{2}\right)^{2}}$

and

$$
\begin{aligned}
\Delta U^{R}(\gamma \rightarrow 1)= & -\frac{1}{2} \frac{r v A}{\left(r \sigma^{2}\left(\lambda^{2}-1\right)-1\right)^{2}\left(1+r \sigma^{2}\right)\left(-\left(1+r \sigma^{2}\left(1-\lambda^{2}\right)\right)-r v^{2}\right)^{2}} \\
& +\frac{1}{2} \frac{\left(r \sigma^{2}\left(\lambda^{2}-1\right)-1-\lambda-r v^{2}\right)^{2}}{\left(1+r \sigma^{2}\right)\left(r \sigma^{2}\left(\lambda^{2}-1\right)-1-r v^{2}\right)^{2}}
\end{aligned}
$$

with

$$
\begin{aligned}
A= & \sigma^{2} r\left[\left(4 \lambda^{2}+2 \lambda-3\right)+r^{2} \sigma^{4}\left(2 \lambda^{5}-\lambda^{4}-4 \lambda^{3}+2 \lambda^{2}+2 \lambda-1\right)\right. \\
& \left.+r \sigma^{2}\left(-3 \lambda^{4}-4 \lambda^{3}+6 \lambda^{2}+4 \lambda-3\right)\right]+v^{2} \sigma^{2} r^{2}\left[r \sigma^{2}\left(\lambda^{2}-2 \lambda^{3}+2 \lambda-1\right)\right. \\
& \left.+2\left(\lambda^{2}+\lambda-1\right)\right]-r v^{2}-1
\end{aligned}
$$

For $\lambda<0 \quad A<0$ holds such that $\Delta U^{R}(\gamma \rightarrow 1)>0$. Only for $-\left(1+r \sigma^{2}\right)\left((1+\lambda)^{2}+r \sigma^{2}(1+2 \lambda)\right)>r v^{2}\left[2 r v^{2}+(3+2 \lambda)\left(1+r \sigma^{2}\right)\right]$, $\Delta U^{R}(\gamma \rightarrow 0)<0$. Then, from the intermediate value theorem it follows that there exists a critical value $\hat{\gamma}^{R} \in(0,1)$ such that $\Delta U^{R}\left(\hat{\gamma}^{R}\right)=0$. Notice that

$$
\frac{\partial \Delta U^{R}}{\partial v}=\frac{-r v\left(1-2 \lambda^{2}-2 \lambda+r v^{2}(1-2 \lambda)+r \sigma^{2}(1-2 \lambda)\left(1-\gamma \lambda^{2}\right)\right)}{-\left(r \sigma^{2}\left(1-\gamma \lambda^{2}\right)+1+r v^{2}\right)^{3}}>0
$$

\footnotetext{
12 As mentioned in Sect. $4.2 \gamma=0$ and $\gamma=1$ are knife-edge cases under $A_{2}$ such that we consider $\gamma=0$ and $\gamma=1$ except for $s^{R * *}$ where we consider the limits for $\gamma \rightarrow 0$ and $\gamma \rightarrow 1$, respectively.
} 
and

$$
\frac{\partial\left(\frac{\partial \Delta U^{R}}{\partial v}\right)}{\partial \gamma}=\frac{2 r^{2} v \lambda^{2} \sigma^{2}\left\{1-3 \lambda^{2}-2 \lambda+r v^{2}(1-2 \lambda)+r \sigma^{2}(1-2 \lambda)\left(1-\gamma \lambda^{2}\right)\right\}}{\left(r \sigma^{2}\left(1-\gamma \lambda^{2}\right)+1+r v^{2}\right)^{4}}>0 .
$$

Thus, $\Delta U^{R}$ is monotonically increasing in $v$ and this effect is monotonically increasing in $\gamma$. Hence, if $\hat{\gamma}^{R}$ exists it is unique such that $U^{R *} \leq U^{R * *}$ if $\gamma \frac{<}{>} \hat{\gamma}^{R}$. Furthermore, it follows that $\frac{\partial \gamma^{R}}{\partial v}<0$. It also follows that if $\Delta U^{R}(\gamma \rightarrow 0)>0$ no $\hat{\gamma}^{R}$ exists such that $A_{1}$ strictly dominates $A_{2}$ for all $\gamma \in(0,1)$.

Proof of Proposition 3 From the proof of Proposition 2 we know $\Delta U^{R}(\gamma \rightarrow 0)$ and $\Delta U^{R}(\gamma \rightarrow 1)$.

$\Delta U^{R}(\gamma \rightarrow 0)>0$ for $\lambda>0$. For $\lambda \leq 0 \Delta U^{R}(\gamma \rightarrow 1)>0$ holds true. Thus, $\Delta U^{R}(\gamma \rightarrow 1)<0$ requires $\lambda>0 . \Delta U^{R}(\gamma \rightarrow 1)$ can be alternatively written as

$$
\Delta U^{R}(\gamma \rightarrow 1)=\frac{1}{2} \frac{c_{1}+c_{2}+c_{3}}{\left(r \sigma^{2}\left(\lambda^{2}-1\right)-1\right)^{2}\left(1+r \sigma^{2}\right)\left(-\left(1+r \sigma^{2}\left(1-\lambda^{2}\right)\right)-r v^{2}\right)^{2}}
$$

with

$$
\begin{gathered}
c_{1}=(1+\lambda)^{2}\left(r \sigma^{2}\left(\lambda^{2}-1\right)-1\right)^{2}\left(r \sigma^{2}(\lambda-1)-1\right)^{2}>0 \\
c_{2}=-r v^{2}\left(r \sigma^{2}\left(\lambda^{2}-1\right)-1\right)\left(c_{21}+c_{22}+c_{23}\right)
\end{gathered}
$$

with $c_{21}=r^{2} \sigma^{4}(\lambda-1)(1+\lambda)\left(2 \lambda^{2}+2 \lambda-3\right)$

$$
\begin{gathered}
c_{22}=-r \sigma^{2}\left(2 \lambda^{3}+7 \lambda^{2}-6-3 r \sigma^{2}\right) \\
c_{23}=3+2 \lambda \\
c_{3}=r^{2} v^{4}\left[(\lambda-1)(1+\lambda)\left(\lambda^{2}+2 \lambda-2\right) r^{2} \sigma^{4}-2 r \sigma^{2}\left(2 \lambda^{2}+\lambda-2\right)+2\right] .
\end{gathered}
$$

For $\lambda \leq \sqrt{3}-1 \quad c_{1}, c_{2}$, and $c_{3}>0$ such that $\Delta U^{R}(\gamma \rightarrow 1)>0$. Thus, $\Delta U^{R}(\gamma=1)<0$ requires $\lambda>\sqrt{3}-1 \approx 0.732$. If $\lambda$ marginally exceeds $\sqrt{3}-1$ the first term $(\lambda-1)(1+\lambda)\left(\lambda^{2}+2 \lambda-2\right) r^{2} \sigma^{4}$ in $c_{3}$ becomes negative while $-2 r \sigma^{2}\left(2 \lambda^{2}+\lambda-2\right)+2$ is still positive as is $c_{2}$. Thus, if $\sigma$ is sufficiently high and $v$ sufficiently high $\Delta U^{R}(\gamma \rightarrow 1)<0$ results. If $\lambda$ increases further $c_{3}$ and $c_{2}$ are 
positive, independently of the value of $\sigma$. If $v$ is sufficiently high $\Delta U^{R}(\gamma \rightarrow 1)<0$ holds true.

Thus, if this condition is fulfilled $\Delta U^{R}(\gamma \rightarrow 0)>0$ and $\Delta U^{R}(\gamma \rightarrow 1)<0$ hold true such that (due to the intermediate value theorem) there exists a critical value $\hat{\gamma}^{R} \in(0,1)$ such that $\Delta U^{R}\left(\hat{\gamma}^{R}\right)=0$. From the proof of Proposition 2 we know $\frac{\partial \Delta U^{R}}{\partial v}$ and $\frac{\partial\left(\frac{\partial \Delta U^{R}}{\partial v}\right)}{\partial \gamma}$. For $\lambda>\sqrt{3}-1 \frac{\partial \Delta U^{R}}{\partial v}<0, \frac{\partial\left(\frac{\partial \Delta U^{R}}{\partial v}\right)}{\partial \gamma}<0$ hold true such that the critical value $\hat{\gamma}^{R}$ is unique.

\section{References}

Alexander, David, and Christopher Nobes. 2013. Financial Accounting-An International Introduction. 5th ed. Harlow: Pearson Education.

Andre, Paul, and Andrei Filip. 2012. Accounting conservatism in europe and the impact of mandatory IFRS adoption: Do country, institutional and legal differences survive? Working Paper Essec Business School.

Antle, Rick, and Joel S. Demski. 1989. Revenue recognition. Contemporary Accounting Research 5 (2): 423-451.

Christensen, Peter O., Gerald A. Feltham, and Florin Şabac. 2003. Dynamic incentives and responsibility accounting: a comment. Journal of Accounting and Economics 35 (3): 423-436.

Christensen, Peter O., Gerald A. Feltham, and Florin Şabac. 2005. A contracting perspective on earnings quality. Journal of Accounting and Economics 39 (2): 265-294.

DeGroot, Morris H. 1970. Optimal Statistical Decisions. New Jersey: Wiley.

Dutta, Sunil, and Xiao-Jun Zhang. 2002. Revenue recognition in a multiperiod agency setting. Journal of Accounting Research 40 (1): 67-83.

Dye, Ronald A., and Sri S. Sridhar. 2004. Reliability-relevance trade-offs and the efficiency of aggregation. Journal of Accounting Research 42 (1): 51-88.

Feltham, Gerald A., and Jim Xie. 1994. Performance measure congruity and diversity in multi-task principal/agent relations. The Accounting Review 69 (3): 429-453.

Fudenberg, Drew, Bengt Holmström, and Paul Milgrom. 1990. Short-term contracts and long-term agency relationships. Journal of Economic Theory 51 (1): 1-31.

Gibbons, Robert. 2005. Incentives between firms (and within). Management Science 51 (1): 2-17.

Gjesdal, Froystein. 1981. Accounting for stewardship. Journal of Accounting Research 19 (1): 208-231.

Indjejikian, Raffi, and Dhananjay Nanda. 1999. Dynamic incentives and responsibility accounting. Journal of Accounting and Economics 27 (2): 177-201.

Liang, Lihong, and Edward Riedl. 2014. The effect of fair value versus historical cost reporting model on analyst forecast accuracy. The Accounting Review 89 (3): 1151-1177.

Pacter, Paul. 2015. The global reach of IFRS is expanding. http://www.ifrs.org/Features/Pages/Globalreach-of-IFRS-is-expanding.aspx. 24 Feb 2017.

Schöndube-Pirchegger, Barbara, and Jens Robert Schöndube. 2012. The value of extended delegation in dynamic agency. Management Accounting Research 23 (3): 158-170.

Schöndube, Jens Robert. 2008. Early versus late effort in dynamic agencies with unverifiable information. Business Research 1 (2): 165-186.

Zhang, Xiao-Jun. 2012. Information relevance, reliability and disclosure. Review of Accounting Studies 17 (1): 189-226. 